Tropical Journal of Pharmaceutical Research April 2013; 12 (2): 255-264

ISSN: $1596-5996$ (print); 1596-9827 (electronic)

(C) Pharmacotherapy Group, Faculty of Pharmacy, University of Benin, Benin City, 300001 Nigeria.

All rights reserved.

Available online at http://www.tjpr.org

Review Article

http://dx.doi.org/10.4314/tjpr.v12i2.19

\title{
Effect of Zeta Potential on the Properties of Nano-Drug Delivery Systems - A Review (Part 1)
}

\author{
Soheyla Honary* and Foruhe Zahir \\ Mazandaran University of Medical Sciences, School of Pharmacy, Sari, Iran \\ *For correspondence: E-mail: shonary@mazums.ac.ir, shonary@yahoo.com
}

Received: 17 August 2012

Revised accepted: 3 January 2013

\begin{abstract}
Zeta potential is a scientific term for electrokinetic potential in colloidal systems which has a major effect on the various properties of nano-drug delivery systems. Presently, colloidal nano-carriers are growing at a remarkable rate owing to their strong potential for overcoming old challenges such as poor drug solubility and bioavailability. Furthermore, they show an unlimited capacity in the field of drug targeting. The properties of nano-medicines such as release from dosage forms at specific sites as well as drug circulation and absorption into body membranes are dramatically affected by some physical and chemical characteristics of nano-drugs. Particle size and charge are two major factors which could play key roles in this regard. In this paper, the effect of zeta potential on different properties of nano-drug delivery systems is reviewed.
\end{abstract}

Keywords: Nano-drug delivery, Zeta potential, Drug targeting, Particle size, Particle charge

Tropical Journal of Pharmaceutical Research is indexed by Science Citation Index (SciSearch), Scopus, International Pharmaceutical Abstract, Chemical Abstracts, Embase, Index Copernicus, EBSCO, African Index Medicus, JournalSeek, Journal Citation Reports/Science Edition, Directory of Open Access Journals (DOAJ), African Journal Online, Bioline International, Open-J-Gate and Pharmacy Abstracts

\section{INTRODUCTION}

\section{Zeta potential (ZP)}

Zeta potential is a scientific term for electrokinetic potential in colloidal systems, i.e., electric potential in the interfacial double layer at the location of the slipping plane versus a point in the bulk fluid away from the interface [1]. This term expresses the potential difference between the dispersion medium and the stationary layer of fluid attached to the dispersed particle. Although zeta potential is not equal to the Stern potential or electric surface potential in the double layer, it is often the only available path for the characterization of double-layer properties [1].

Coulomb interactions are the strongest physical forces between any two objects. Electrokinetic or $\zeta$-potential is defined as the average electrostatic potential existing at the hydrodynamic plane of shear, somewhere between the Stern plane and the end of the diffuse layer, normally considered to be $0.2 \mathrm{~nm}$ from the surface $[2,3]$. On the other hand, the electric double layer formed at the boundary between a solid surface and an electrolyte solution determines its electrokinetic (interfacial double layer or charge) properties. Thus, zeta potential can be defined as the electrokinetic value associating a realistic magnitude of surface charge [4-9]. Measurement of $\zeta$-potential is currently the simplest and most straightforward way to characterize the surface of charged colloids, and conclusions are easily drawn from the analysis of its data regarding concentration, distribution, adsorption, ionisation, exposure or shielding of charged moieties [3]; its unit is usually milivolt [7]. 
Zeta potential can affect the pharmacokinetic properties of nanosystems in the body $[7,10]$ or may affect the phagocytosis of the nanoparticles in the blood stream [11-13]. Nanoparticles can be detected by electrostatic methods, by condensation on particles to grow them until they are measurable optically, or by other methods. Electrostatic methods require that the particles be charged and are not very sensitive [14].To fully characterize the charge conditions of particles, zeta potential measurements should be performed in distilled water and in the original dispersion medium of the suspension. Very often, ZP measurements are performed in buffers of varying molarities, physiological salt solution (some pharmacists think being physiological is a priori good) or other media. These measurements are rather meaningless for determining surface potential [15] or physical long-term stability under these measuring conditions [16].

\section{Measurement of ZP}

Although ZP is not measurable directly, it can be calculated using theoretical models. Electrokinetic phenomena and electroacoustic phenomena are the usual sources of data for calculation of ZP. The ZP of a dispersion is measured by applying an electric field across the dispersion [17]. Particles within the dispersion with a ZP will migrate toward the electrode of opposite charge with a velocity proportional to the magnitude of the zeta potential. There are two different experimental techniques: microelectrophoresis which has the advantage of yielding an image of the moving particles, and electrophoretic light scattering which is based on dynamic light scattering. The latter can be used to characterize very small particles. The frequency shift or phase shift of an incident laser beam caused by these moving particles is measured as the particle mobility, and this mobility is converted to ZP using Smoluchowski or Huckel theory. Both of these methods may require dilution of the sample. Sometimes, this dilution might affect the properties of the sample and change ZP. Sample dilution using equilibrium supernatant is the best justified way to perform this dilution. Equilibrium supernatant is readily obtained by centrifugation [17-18].

\section{ZP AND TARGETED DRUG DELIVERY}

Nanoparticle surface is a very important consideration in targeting drug delivery. Indeed, once in the blood stream, conventional nanoparticles (no surface medication) and negatively charged particles can be rapidly opsonized and massively cleared by fixed macrophages. It is well known that the reticuloendothelial system (RES), mainly the liver and spleen, is a major obstacle to active targeting because of its ability to recognize these systems, remove them from systemic circulation, and consequently, avoid the effective delivery of the nano drug to organs other than those of the RES. Surface modification of these polymer nanoparticulate systems with hydrophilic polymers is the most common way to control the opsonization process and to improve the surface properties, especially surface charge, of the system [19].

\section{Effect of ZP on cellular uptake}

Physicochemical properties, such as particle size, shape and surface charge, play a key role in the cellular uptake of nanoparticles. The uptake of nanoparticles by cells can be viewed as a two step process: first, a binding step on the cell membrane and second, the internalization step [20]. The attachment of nanoparticles to cell membrane seems to be most affected by the surface charge of the particles [21-22]. Variation of the particle surface charge could potentially control binding to the tissue and direct NPs to cellular compartments both in vitro and in vivo. Cellular surfaces are dominated by negatively charged sulphated proteoglycans moleculs that play pivotal roles in cellular proliferation, migration, and motility [23-24]. Cell surface proteoglyans consist of a core protein anchored to the membrane and linked to one or more glycosaminoglycan side chains (heparan, dermatan, keratan or chondrotine sulfates) to produce a structure that extends away from the cell surface.

Glycosaminoglycans are highly anionic, and interaction between proteoglycans and NP shells, if positively charged, tend to be largely ionic [25]. Once inside the cell, degradation of polymers may occur, but targeting specific intracellular organelles is possible depending on the surface charge and attached ligands [26]. Nanoparticles with higher surface charge bound strongly to the cell membrane and showed a higher cellular uptake, where electrostatic interactions between the anionic membrane and cationic nanoparticles facilitate the uptake. After the adsorption of the nanoparticles on the cellular membrane, the uptake occurs via several possible mechanisms such as pinocytosis, nonspecific or receptor-mediated endocytosis or phagocytosis [27-28].

The uptake of cerium oxide nanoparticles as a function of the surface charge of the particles was studied by Patil et al. Their results showed 
that nanoparticles with ZP of - $43 \mathrm{mV}$ have the highest cellular uptake compared with other formulations with less negative charge and/or positive surface charge. Nanoparticles show a high affinity for cellular membrane mainly due to electrostatic interactions [23]. It is already known that cell membranes have large negatively charged domains, which should repel negatively charged nanoparticles. The previous study on A549 cells showed that these cells indicated mean ZP of $-10.2 \mathrm{mV}$ with deviation of $\pm 19.7 \mathrm{mV}$. The authors concluded that in that case, there are few cationic sites for adsorption of negatively charged particles. Previous literature suggested that the negatively charged particles bind at the cationic sites in the form of clusters [21] because of their repulsive interactions with the large negatively charge domains of cell surface. Moreover, the nanoparticles, already bound on the cell surface present a reduced charge density that may favor adsorption of other free particles. Totally, it seems, the high cellular uptake of negatively charged nanoparticles in their study, is related first to the non-specific process of nanoparticles adsorption on the cell membrane and second to formation of nanoparticle clusters. Studies by Limbach et al. on human lung fibroblast cells also indicated that cells rapidly adsorb negatively charged ceria nanoparticles [29]. The adsorption of the negatively charged particles at the positively charged sites via electrostatic interaction can lead to localized neutralization and a subsequent bending of the membrane favoring in turn endocytosis for cellular uptake [30]. Thus the formulation of nanoparticles with different surface properties can influence their cellular uptake and intracellular distribution [31-32] and it is possible to localize the nanoparticles to specific intracellular targets (lysosomes, mitochondria, cytoplasm and etc) by modifying their surface charge [21].

There are some investigations that show the effect of surface charge in this regard. For example polymer charge density of dendrimers was found to significantly impact membrane permeability. The most densely charged polymer facilitates the transport of dye molecule across the membrane [33]. Other investigation showed that lipid coating of ionically charged nano particles was able to increase endothelial cell layer crossing 3 or 4 fold compared with uncoated particles, whereas nanoparticles coating of neutral particles did not significantly alter their permeation characteristics across the endothelial cell monolayer [34].

In an interesting investigation, histidine was partially substituted in the amino group of glycol chitosan self-assembled nanoparticles. Since histidine is strongly cationic at acidic $\mathrm{pH}$, it interacts with negatively charged endosomal membranes, induces influx. The efficiency of drug delivery was significantly enhanced probably due to this interaction and the destabilization of endosomal membrane (Fig 1) [35]. Following cellular uptake, nanoparticles are transported to primary endosomes and to sorting endosomes. A fraction of nanoparticles can be recycled back to the exterior of cell by mean of exocytosis, while the rest reaches secondary endosomes. Secondary endosomes fuse with lysosomes to form endo-lysosome and the efficiency of cytoplasmic delivery is governed by the ease and rapidity of escape of nanoparticles from endo-lysosome. Previous literatures proposed and confirmed that the surface charge reversal of nanoparticles in the acidic $\mathrm{pH}$ of endo-lysosomes was responsible for the endolysosomal escape of nanoparticles. Therefore, surface modification of nanoparticles can be attempted in order to influence targeting of drugs either to endo-lysosomes or to cytoplasm [36].

\section{Effect of ZP on skin drug delivery systems}

Transdermal drug administration systems have been limited to drugs with the right combination of molecular weight, lipophilicity and charge [36]. Cationic compounds can also have a positive effect on skin permeation, since the skin carriers a negative surface charge due to phosphatidyl choline [37] and carbohydrates [38] found in mammalian cells and contain negatively charged groups [39]. Percutaneous penetration implies several steps. The release of a drug through skin will depend on the physicochemical properties of the drug itself combined with the influence of the vehicle to alter the drug penetration profile [40]. The positively charged nanoemulsions containing phytosphingosine (PS) were found to be more effective in terms of skin diffusion of fludrocortisones acetate and flumethasone pivalate through porcine skin than the negatively charged ones [41]. As mentioned, the interaction of nanoemulsions with skin depends upon a number of factors including also the electrical charge of the droplets. The results obtained suggested that positively charged particles of the nanoemulsion systems are able to carry efficiently fludrocortisone acetate and flumethasone pivalate into the skin and subsequently promote the penetration of the drugs through skin. The degree of skin binding is probably more important with the positively charged particles than with the negatively one as it is known that the skin is negatively charged at neutral $\mathrm{pH}$. 


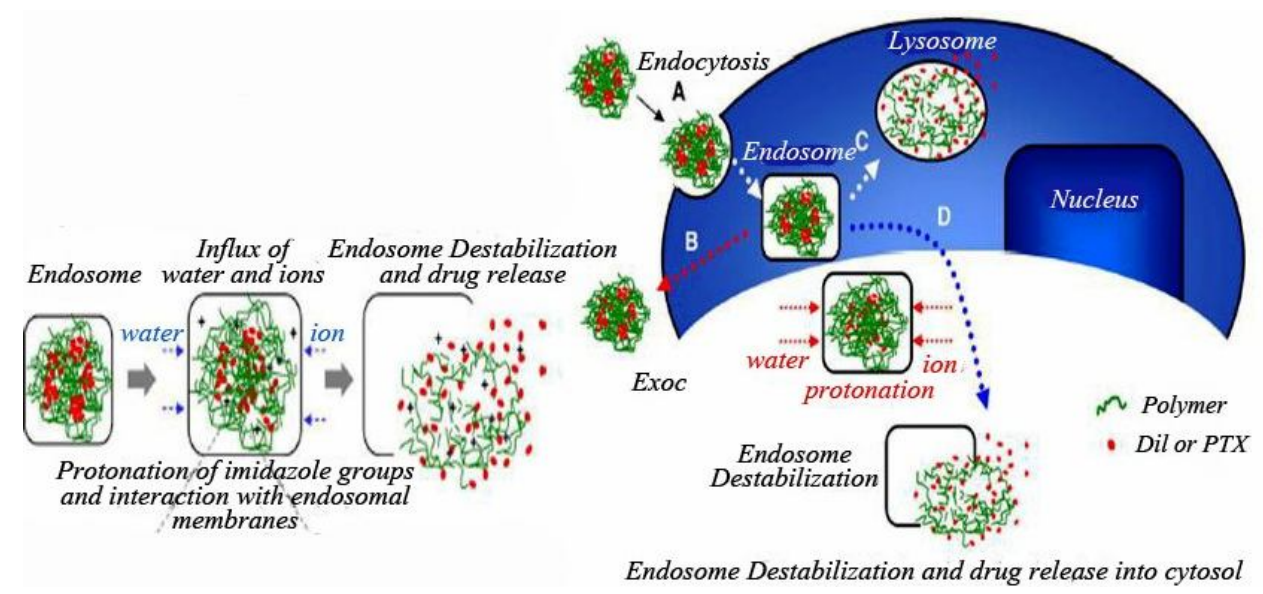

Figure 1: Destabilization of endosomal membrane due to charge interaction [35]

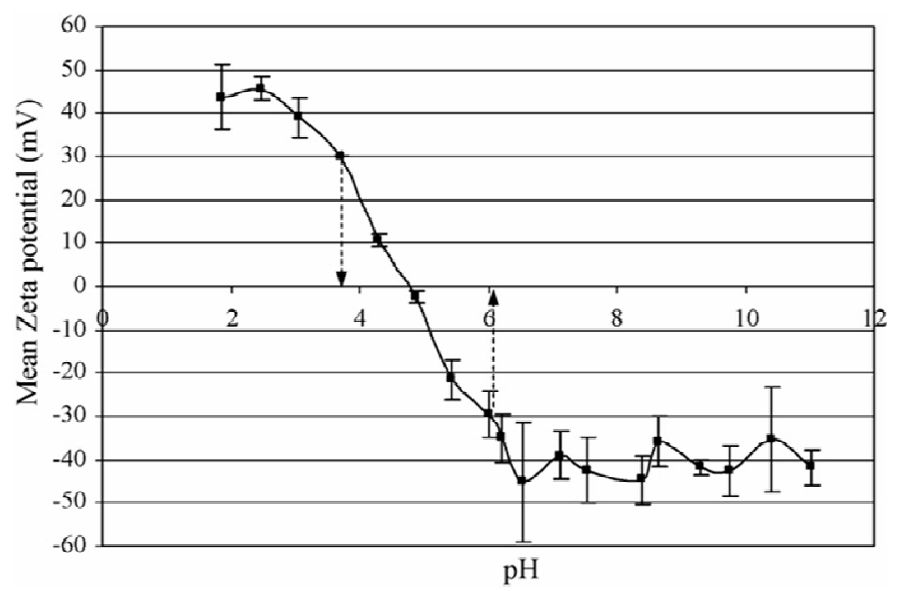

Figure 2: The zeta potential of an aqueous suspension of nanospheres with $\mathrm{pH}$ values adjusted between 2 and 12. Dashed arrows indicate the $\mathrm{pH}$ range between which the zeta potential of the nanoparticle formulation falls between +30 and $-30 \mathrm{mV}[42]$.

A novel transdermal delivery device, microneedle, mediated for delivery of nanoparticles into human skin, was studied on the permeation of nanoparticles through microchannels created within the skin surface. The zeta potential of nanoparticle formulations was measured at discriminate $\mathrm{pH}$ values between 2 and 12. Fig. 2 illustrated the direct influence of $\mathrm{pH}$ on the zeta potential of a colloidal particle and from this data an isoelectric point (the $\mathrm{pH}$ value of a colloidal formulation at which the zeta potential becomes $0 \mathrm{mV}$ ) of approximately $\mathrm{pH} 5$ was calculated. In simple terms, an isoelectric value of $\mathrm{pH} 5$ means that within this particular aqueous environment hydrogen ions are required to neutralise what must be a dominant negative charge on the surface of the nanoparticle. Therefore, although the nanoparticles have been functionalized to include reactive amine groups, it is negatively charged sulphate groups (created on the surface of the nanoparticle during the polymerization process) which contribute more significantly to the surface potential of the particle. The ability to reverse the zeta potential of nanoparticles, by adjustment of $\mathrm{pH}$, was utilised to investigate the influence of a particles surface potential on its permeation through microconduits. It was shown that at $\mathrm{pH}$ of 7.4 (physiological $\mathrm{pH}$ ) fluorescent nanospheres possessed a negative zeta potential $(-40 \mathrm{mV})$ whilst at $\mathrm{pH} 3$ the surface potential of fluorescent nanospheres was reversed, i.e. the zeta potential was approximately $+40 \mathrm{mV}$. However the $\mathrm{pH}$ of the receptor phase in all Franz-type diffusion cells was measured both before and after each experiment and indicated that the desired $\mathrm{pH}$ was maintained for the duration of the experiment. At pH 7.4, where both the nanospheres and the membrane surface possess negative zeta potentials, diffusion through $10 \mu \mathrm{m}$ pores occurred rapidly. After $4 \mathrm{~h}$ almost $80 \%$ of the applied formulation was detected in the receptor phase. At this stage, the concentration of nanoparticles is approaching equivalence across the membrane, suggesting that permeation in this model is a simple diffusive process and that equilibrium can be reached in a relatively short period of time (Fig 2) [42]. 


\section{Effect of ZP on tumor cells targeting}

Cell surfaces, especially cancer cell surfaces, are usually charged negatively due to the translocation of negatively charged constituents of the inner layer of the cell membrane (e.g., phosphatidylserine, anionic phospholipids, glycoproteinsand proteoglycans) to the cell surfaces in the case of cancers [43]. The increased uptake of chitosan-modified paclitaxelloaded PLGA nanoparticles (C-NPs) into the cell at lower $\mathrm{pH}$ medium appears to be attributable in part to the electrostatic interaction between the positive charge of C-NPs and negative charge of the cancer cell surfaces [44]. Evidence accumulated during the past 50 years shows that the $\mathrm{pH}$ of most solid tumors in patients ranges from 5.7 to 7.8 with a mean value of 7.0. Moreover, greater than $80 \%$ of these measured values are smaller than $\mathrm{pH} 7.2$ [45]. Tumor tissues contain large, acid-outside plasma $\mathrm{pH}$ gradients, while normal tissues generally have alkaline-outside $\mathrm{pH}$ gradients [46]. Therefore, the extracellular $\mathrm{pH}$ of malignant tumors is significantly lower than that of normal tissues under physiological conditions [47]. As the $\mathrm{pH}$ became acidic, the zeta potential of NPs became positive, which enhanced the electrostatic interaction between the C-NPs and tumor tissues. In addition, negative charges on the endothelial cell membranes of the tumor vasculature might have potentiated the electrostatic interaction between positively charged nanoparticles and tumors. Thus, these two factors - more positive charges of C-NPs at the tumor site and more negative charges of tumor cells/vasculature - appear to be responsible for the tumor-specific accumulation of C-NPs. This method used to accelerate in vitro uptake of coumarin 6 to cancer cells, enhanced cytotoxicity of paclitaxel and increased in vivo accumulation of coumarin 6 in tumor-bearing lungs [48].

Cationic liposomal doxorubicin (LPs-DOX) and paclitaxel (LPs-PTX) were used for targeting the Fibroblast growth factor receptors (FGFRs) which are overexpressed on the surface of a variety of tumor cells. Biodistribution study in B16 tumor-bearing mice showed that truncated human basic fibroblast growth factor peptide which was attached to the surface of cationic liposomal paclitaxel (tbFGF-LPs-PTX) achieved 7.1-fold accumulation of paclitaxel in tumor tissue than those of free paclitaxel [49]. The anionic diblock copolymer of polymethacryloyl sulfadimethoxine (PSD) was complexed with cationic TAT of the micelles to achieve the final carrier, which could systemically shield the micelles and expose them at slightly acidic tumor
$\mathrm{pH}$. Zeta potential measurements showed a trend substantiating the shielding/deshielding process. Flow cytometry and confocal microscopy showed significantly higher uptake of TAT micelles at $\mathrm{pH} 6.6$ compared to $\mathrm{pH} 7.4$, indicating shielding at normal $\mathrm{pH}$ and deshielding at tumor $\mathrm{pH}$. Confocal microscopy indicated that the TAT not only translocates into the cells but is also seen on the surface of the nucleus. From $\mathrm{pH}$ 8.0 to 6.8 the zeta potential was around zero indicating complete shielding of TAT and from 6.6 to 6.0 the zeta potential was increased to 6.0 $\mathrm{mV}$, which is the same as the zeta potential measured for TAT micelle alone, implicating the de-shielded TAT. The TAT peptide has high positive charge still the zeta potential of the micelles is low, this is attributed to the low surface density of TAT conjugated to the micelle surface [50].

Drug delivery systems may bring us a step closer to a safe and universal targeting system for acidic solid tumors. The vascular permeability is limited by the size and charge of the drug as well as the structures of the vessel wall particularly the basement membrane [51]. Direct access to intravenously administered agent, rapid proliferated of endothelial cells and over expression of negatively charged functional groups along vessels are potentially exploitable feature of tumors [51-52].

Nanoparticleas with positive charge are preferentially taken up by the tumors [53-54]. and retained for a longer time span compared to negatively charge or neutral particles [53] because phosphatidyl serine, a negatively charge residue, is translocated to the surface of the cancer cells [59] and nanoparticles with positive charge can translocated by the tumor cells through either fluid phase endocytosis, or charge interactions and ligand receptor docking [55]. Cationic drug carriers can also be used to enhance the interaction of anti-angiogenic agents with integrins. Integrins are involved in cell invasion and metastasis and in signaling processes [38]. This strategy to target the tumor cells is named passive targeting or the enhanced permeability and retention (EPR) effect wherein carriers of appropriate particle size, charge and stability will preferentially accumulate in tumors [56]. Investigations show that cationic liposomes bind by electrostatic interactions to negatively charge surface in tumor endothelial cells [57]. Increasing the charge of cationic PEGylated liposomes decrease their accumulation in the spleen and blood while increasing their uptake by the liver and an increasing in the accumulation of liposomes in tumor vessels [58]. Moreover, the presence of charged functionalities on the 
nanoparticles surface may increase nonspeciffic uptake and making the preparation less effective in targeting, Because negative charge is available on other tissues too [34]. For example positive nanoparticles can interact with negative plasma proteins and form nanoparticles-proteins aggregates [54].

\section{Effect of ZPs on brain targeting}

Transportation of nanoparticles from the BBB is involving electrostatic interaction between a positively charged ligand and the negatively charged membrane of cells at the BBB that is named adsorptive mediated endocytosis (AME) [60]. Positive zeta potensial on nanoparticles can help the transportation of nanoparticles from BBB. The anionic sites were located on the luminal surface of brain capillaries is due to the sialic acid residues of glycoproteins. AME of nanoparticles can accurs in other ways like choline transporter in the brain. These carriers are consisting of anionic sites which interact with positively charged quaternary ammonium group [57].

There are some established works in the delivery of positive proteins or positive nanoparticles to the brain. For example cationazed albumin nanoparticles [35] or immunogolubins [57] were efficiently transport across the BBB. The other example is the etoposide-loaded positively charged tripalmitin nanoparticles that achieve highest brain concentration when compared to negatively charged etoposide incorporated tripalmitin nanoparticles and etoposide solution [61].

Methoxy PEG-PLA and maleimide PEG-PLA were synthesized via ring opening polymerization of L-lactide and used to prepare PEGylated nanoparticles (NPs) loaded with sulpiride by emulsification and solvent evaporation method. Thiolated cationized bovine serum albumin (CBSA) was conjugated through the maleimide function to the NPs. Characterized particles (mean particle size $329 \pm 44 \mathrm{~nm}$ ) indicated the conjugation of cationic albumin to NPs (zeta potential shift from $-39 \mathrm{mV}$ to $-19 \mathrm{mV}$ ). Fluorescence showed a high accumulation of CBSA-NPs in brain compared to that of BSANPs and uncoated NPs supported by plasma and urine profile. ZP represents the difference in the electrical charge between the dense layers of ions that surrounds the particle and the charge of the bulk of the suspended fluid surrounding the particles.

The ZP of CBSA-NPs can be attributed to two factors; first, the PEG surface shielding effect on offsetting the negative charge of the PLA segments in the NPs core and second, the CBSA cationization degree. The ZP measured for uncoated NPs and CBSA-NPs were around -39 $\mathrm{mV}$ and $-19 \mathrm{mV}$, respectively. The potential difference in ZP values represents successful attachment of CBSA to NPs and change in surface charge property of the particle. Although ZP for the obtained particle was towards negative side, it supported the hypothesis that overall, at the local microenvironment surrounding each CBSA milieu on the NPs surface, the net electrical charge would be positive and facilitating CBSA at periphery of the NPs to electrostatically bind the negatively charged residues on the endothelial cells of BBB. Therefore, it could be assumed that due to relatively less negative surface charge of the particle, it gets attracted and adsorbed to the BBB endothelial cells and thus enhances the availability (Fig 3) [62].

The procationic liposomes (PCLs) were neutral or negatively charged at physiological $\mathrm{pH}$, and when they touched brain capillary endothelial cells with the help of a brain-targeting ligand, lactoferrin (Lf), they were changed into cationic liposomes (CL). The PCLs and lactoferrinmodified procationic liposomes (Lf-PCLs) with different CHETA/Lf ratio were prepared and characterized in previous literatures. The primary brain capillary endothelial cells (BCECs) were cultured to investigate the potential cytotoxicity and uptake of liposomes in vitro. An in vitro model of the blood-brain barrier (BBB), developed by the co-culture of BCECs and astrocytes
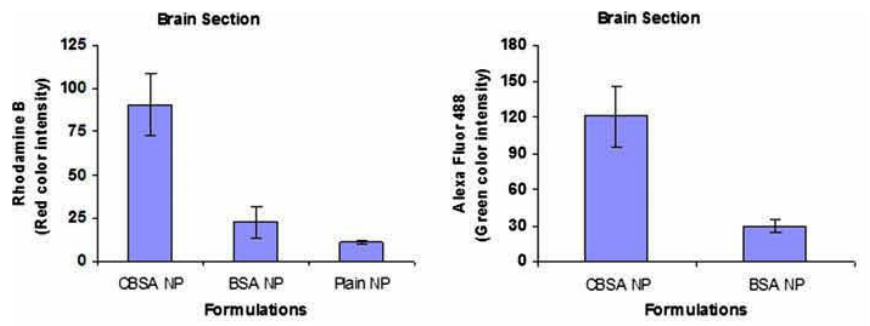

Figure 3' The effect of zeta potential on brain targeting of nanoparticles [62].

(ACs), was employed to evaluate the ability and mechanisms of liposomes to cross endothelial cells. The liposome uptake by the mouse brain in vivo was detected by HPLC-fluorescence analysis. The results indicated that compared with the conventional liposomes and CLs, PCL and Lf-PCLs showed an improved performance in the uptake efficiency and cytotoxicity. Besides the uptake mediated by clathrin-dependent endocytosis of PCL, Lf-PCL crossed the BCECs through lipid raft/caveloae-mediated endocytosis. 
The endocytosis involved in the transport of LfPCL crossing BBB was mediated by both receptor- and absorption-mediated transcytosis compared with the conventional liposomes. The zeta potential of coumarin- 6 loaded PCLs and LfPCLs were from -32.3 to $-4.3 \mathrm{mV}$. And it was shown that the positively charged Lfs might have attached onto the surface of the PCLs. The comparison of zeta-potential of PCLs and LfPCLs further confirmed the interaction of Lfs with PCLs [63].

The BBB penetration and distribution of PEGylated PFMSNs and FMSNs in rat brain were investigated not only at the cellular level with Confocal laser scanning microscopy (CLSM), but also at the subcellular level with transmission electron microscopy (TEM). The results provide direct evidence that PEGylated PFMSNs could penetrate the BBB and spread into the brain parenchyma. According to the values of $f$-potential, the surface charges of FMSNs changed from negative $(-22.43 \mathrm{mV})$ to positive $(+18.93 \mathrm{mV})$ as a result of PAMAM conjugation, then the charge changed to nearly neutral $(+1.49 \mathrm{mV})$, indicating a further modification of PEG [64].

In order to develop a novel brain-targeting delivery system for anti-neuroexcitation peptide (ANEP), a water-soluble cationic chitosan derivative, $\mathrm{N}$-trimethyl chitosan chloride (TMC), was synthesized. The nanoparticles have a positive zeta potential of $30.7 \mathrm{mV}$. Absorptionmediated transcytosis (AMT) is triggered by an electrostatic interaction between the positively charged moiety of the nanoparticles and a negatively charged plasma membrane surface region on the brain capillary endothelium. Fluorescent micrographs also showed the corresponding brain biodistribution results in the four groups [65]. Huang et al. [66] proved that the uptake of chitosan nanoparticles by A549 cells was initiated by electrostatic interactions between the nanoparticles and the cell membrane and Amidi et al. found that FITCalbuminassociated TMC nanoparticles could be transported across the nasal mucosa due to intracellular uptake by epithelial and NALT cells in a series of in vivo uptake studies [67].

\section{Effect of ZP on multi drug resistance (MDR)}

Multi-drug resistance (MDR) of tumor cells is a major obstacle to the success of cancer chemotherapy. MDR is mainly due to the over expression of the plasma membrane Pglycoproteins, which is capable of extruding various generally positively charged xenobiotics including some anticancer drugs. Indeed, to be effective to overcome MDR three criteria need to be fulfilled: 1) particulate structure should adhere to the cell membrane, providing a concentration gradient. 2) The drug release and nanoparticles degradation should occur simultaneously 3 ) an ion pair should form in order to mask the positive charge of the drug. So in this way by masking the ZP in the surface of the nanoparticles could be overcome MDR [52].

Tumor drug resistance significantly limits the success of chemotherapy in the clinic. Tumor cells utilize multiple mechanisms to prevent the accumulation of anticancer drugs at their intracellular site of action. In previous studies the anticancer efficacy of doxorubicin in combination with photodynamic therapy was investigated using methylene blue in a drug-resistant mouse tumor model. Surfactant-polymer hybrid nanoparticles formulated using an anionic surfactant, Aerosol-OT TM (AOT), and a naturally occurring polysaccharide polymer, sodium alginate, were used for synchronized delivery of the two drugs. Their proposed model for AOT alginate nanoparticles - drug-loaded, calciumcrosslinked alginate core, surrounded by one or more bilayers composed of AOT, suggested that only the negative charges present in the core of the nanoparticle matrix (carboxyl groups in alginate) are involved in electrostatic interactions with weakly basic drug molecules. Thus, negative charges (sulfonate of AOT) on the surface of nanoparticles contribute to the negative zeta potential of drug-loaded AOTalginate nanoparticles [68].

The cellular uptake and cytotoxicity of targeted compare with nontargeted doxorubicin (DOX)loaded 9 poly (D,L-lactide co-glycolide) (PLGA) nanoparticle (NP) drug delivery systems in drugresistant ovarian (SKOV-3) and uterine (MESSA/10 Dx5) cancer cell lines. They concluded that cellular uptake and cytotoxicity of DOX can be improved in MES-SA/Dx5 cells by loading DOX into PLGA NPs. The zeta potentials of the DOX-loaded NPs with and without antibody (IgG) conjugation were $-1.3 \pm 3.8 \mathrm{mV}$ and $290-13.2 \pm$ $2.3 \mathrm{mV}$, respectively [69].

Paclitaxel-loaded nanoparticles were prepared by a water-acetone solvent displacement method using commercial PCL and selfsynthesized PCL/Poloxamer 188 compound, respectively. It is also an important factor in determining their interaction with the cell membrane in vivo, which is usually negatively charged. In addition, from the zeta potential data, we can roughly know the dominated component on the particles surface. As shown in Table 1, the zeta potential of PCL/Poloxamer 188 nanoparticles is $-22.77 \mathrm{mV}$ Compared with PCL 
nanoparticles, whose zeta potential is around $35.45 \mathrm{mV}$, a great increase in the absolute value of the PCL/Poloxamer 188 nanoparticle surface charge could be observed. Since Poloxamer 188 is non-ionic, this surface charge increase demonstrated the presence of a Poloxamer 188 layer on the surface, which shifted the shear plane of the diffusive layer to a larger distance [70].

\section{CONCLUDING REMARKS}

Zeta potential is one of important propertied which could play a major role in effectiveness of nanomedicine. Either targeted therapy or stability of dosage forms could be affected by ZP of nano-materials.

\section{REFERENCES}

1. Kutschera HL, Chaoa P, Deshmukha M, Rajana SS, Singha $Y$, Hua $P$, Josephb LB, Steina S, Laskinb $D L$, Sinkoa PJ. Enhanced passive pulmonary targeting and retention of PEGylated rigid microparticles in rats. Int. J. Pharm. 2010; 402: 6471.

2. Khan MK. Nigavekar SS, Minc LD, Kariapper MS, Nair $B M$, Leniak WG, Balogh LP. In vivo biodistribution of dendrimers and dendrimer nanocomposites -implications for cancer imaging and therapy. Technol. Cancer Res. Treat. 2005; 4: 603-613.

3. Rabinovich-Guilatt L, Couvreur P, Lambert G, Goldstein $D$, Benita S,Dubernet $C$. Extensive surface studies help to analyse zeta potential data: the case of cationic emulsions. Chem.phys.lipid. 2004; 131:.113.

4. Chun MS, Cho H, Songb I. Electrokinetic behavior of membrane zeta potential during the filtration of colloidal suspensions. Desalination 2002; 148: 363367.

5. Davis ME, Chen Z, Shin DM. Nanoparticle therapeutics: an emerging treatment modality for cancer. Nat. Rev. Drug Discov. 2008; 7: 771-782.

6. Prokop A, Kozlov E, Carlesso J, Davidson M. Hydrogelbased colloidal polymeric system for protein and drug delivery: physical and chemical characterization permeability control and applications. Adv. Polymer res. 2002; 160: 119173.

7. Huynh NT, Passirani C, Saulnier P, Benoit JP. Lipid nanocapsules, $A$ new platform for nanomedicine. Int. J. Pharm. 2009; 379: 201-209.

8. Singh R, Lillard JR. Nanoparticle-based targeted drug delivery, Experimental and Molecular Pathology. Exp.Mol. Pathol. 2009; 86: 215-223.

9. Agnihotri SA, Mallikavjund NN, Aminabhavi TM. Recent advances on chitosan-based micro and nanoparticles in drug delivery. J. control. Release 2004; 100: 5-28.

10. Jabr-Milane LS, van Vlerken LE, Yadav S, Amiji MM. Multi-functional nanocarriers to overcome tumor drug resistance. Cancer Treat. Rev. 2008; 34: 592602.

11. Shan X, Liu C, Yuan Y, Xu F, Tao X, Sheng Y, Zhou H. In vitro macrophage uptake and in vivo biodistribution of long-circulation nanoparticles with poly (ethylene-glycol)-modified PLA (BAB type) triblock copolymer. Colloids surface $B$ 2009; 72 : 303-311.
12. Panyam J, Zhou WZ, Probha S, Sahoo S, Labhasetwar $V$. Rapid endo-lysosomal escape of poly $(D, L-$ lactide-co-glycolide) nanoparticles: implications for drug and gene delivery. FASEB 2002; 16: 12171226.

13. Langer K, Balthasar S, Vogel V, Dinauer N, Briesen H, Schubert D. Optimization of the preparation process for human serum albumin (HSA) nanoparticles. Int. J. Pharm. 2003; 257: 169-180.

14. León-Rodrigueza L, Leiro-Vidalb J, lanco-Méndeza J, Luzardo-Álvarez A. Incorporation of PVMMA to PLGA MS enhances lectin grafting and their in vitro activity in macrophages. Int. J. Pharm. 2010; 402: 165-174.

15. Radomska-Soukharev A. Stability of lipid excipients in solid lipid nanoparticles. Adv. Drug Deliv. Rev. 2007; 59: 411-418.

16. Mishra PR., Al Shaal L, Müller R.H, Keck CM. Production and characterization of Hesperetin nanosuspensions for dermal delivery. Int. J. Pharm. 2009; 371: 182-189.

17. Butsele KV, Sibreta P, Fustin CA, Gohyb JF, Passirani $C$, Benoitc JP. Synthesis and $\mathrm{pH}$-dependent micellization of diblock copolymer mixtures. J. Colloid. Interf. Sci. 2009; 329: 235- 243.

18. Kedar U, Phutane $P$, Shidhaye S, Kadam V. Advances in polymeric micelles for drug delivery and tumor targeting' Nanomed- Nanotechnol. 2010; 6: 714729.

19. Reis CP, Neufeld RJ, Ribeiro AJ, Veiga $F$. Nanocapsulation I. methods for preparation of drug-loaded polymeric nanoparticles. NanomedNanotechnol. 2006; 2: 8-21.

20. Ciani L, Ristori S, Bonechi C, Rossi C, Martini G. Effect of the preparation procedure on the structural properties of oligonucleotide/cationic liposome complexes (lipoplexes) studied byelectron spin resonance and Zeta potential. Biophys. Chem. 2007; 131: 80-87.

21. Patila S, Sandberg A, Heckert E, Self W, Sea S. Protein adsorption and cellular uptake of cerium oxide nanoparticles as a function of zeta potential. Biomaterials 2007; 28: 4600-4607.

22. Chen Ch, Tsai TH, Huang ZR, Fang JY. Effects of lipophilic emulsifiers on the oral administration of lovastatin from nanostructured lipid carriers: Physicochemical characterization and pharmacokinetics. Eur. J. Pharm. Biopharm. 2010; 74: 474-482.

23. Bernfild M, Gotte M, Park PW, Reizes O, Fitzgerald ML, Lincecum J, Zako M. Functions of cell surface heparan sulphate proteoglycans. Annu. Rev. Biochem. 1999; 68: 729-777.

24. Mislick K A, Baldeschwieler JD. Evidence for the role of proteoglycans in cation-mediated gene transfer. Proc. Natl. Acad. Sci. U.S.A 1996; 93: 1234912354.

25. Panyam $P$, Labhasetwar $V$. Biodegradable nanoparticles for drug and gene delivery to cell and tissue. Adv. Drug. Deliv. Rev. 2003; 55: 329-347.

26. Harting SM, Greene RR, Dikov MM, Prokop A. Davidson $J M$, Multifunctional nanoparticulate polyelectrolyte complexes. Pharm. Res. 2007; 24: 2353-2369.

27. Wilhelma C, Billoteya C, Rogerc JN, Bacria J, Gazeau F. Intracellular uptake of anionic superparamagnetic nanoparticles as a function of their surface coating. Biomaterials 2003; 24: 10011011.

28. Sahay G, Alakhova DY, Kabanov AV. Endocytosis of nanomedicines. J. Control. Release 2010; 145: 182-195.

29. Limbach LK, Li Y, Grass RN, Brunner TJ, Hintermann MA, Muller M. Oxide nanoparticle uptake in human lung fibroblasts: effects of particle size, 
agglomeration, and diffusion at low concentrations. Environ. Sci. Technol. 2005; 39: 9370-9376.

30. Win KY, Feng SS. Effects of particle size and surface coating on cellular uptake of polymeric nanoparticles for oral delivery of anticancer drugs. Biomaterials 2005; 26: 2713-2722.

31. Nam HY. Cellular uptake mechanism and intracellular fate of hydrophobically modified glycol chitosan nanoparticles. J. control. Release 2009; 135: 259267.

32. Cheng $H$, Zeng J, Jing $Y$, Zhang $X$, Zhuo R. Targeted gene delivery mediated by folate-polyethylenimineblock-poly(ethylene glycol) with receptor selectivity. Bioconjugate. Chem. 2009; 20: 481-487.

33. Wolinsky JB, Grinstaff MW. Therapeutic and diagnostic applications of dendrimers for cancer treatment. Adv. Drug Deliver. Rev. 2008; 60: 1037-1055.

34. Kreuter J. Nanoparticulate systems for brain delivery of drugs. Adv. drug deliver. Rev. 2001; 47: 65-81.

35. Park JS, Han TH, Lee KY, Han SS, Hwang JJ, Mon DH, Kim SY, Cho YW. N-acetyl histidine-conjugated glycol chitosan self-assembled nanoparticles for intracytoplasmic delivery of drugs: Endocytosis, exocytosis and drug release. J. Control. Release 2006; 115: 37-45.

36. Staples M, Daniel K, Cima MJ, Langer R. Application of Micro- and Nano-Electromechanical Devices to Drug Delivery. Pharm. Res. 2006; 23: 847-863.

37. Chang JH, Cho MA, Son HH, Lee CK, Yoon MS, Cho $\mathrm{HH}$, Seo DS, Kim KJ. Characterization and formation of phospholipid nanoemulsion coatings on Mg-modified sericite surface. J. Ind. Eng. Chem, 2006;12: 635-638.

38. Robert BCampbell, Positively-charged liposomes for targeting tumor vasculature, Nano technology for cancer therapy, CRC Press, Taylor \& Francis; 2007. 613p.

39. Hoeller S, Sperge, A, Valenta C. Lecithin based nanoemulsions: $A$ comparative study of the influence of non-ionic surfactants and the cationic phytosphingosine on physicochemical behaviour and skin permeation. Int. J. Pharm. 2009; 370: 181-186.

40. Cazares-Delgadillo J, Naik A, Kalia YN, QuintanarGuerrero D, Ganem-Quintanar A. Skin permeation enhancement by sucrose esters: a $\mathrm{pH}$-dependent phenomenon. Int. J. Pharm. 2005; 297: 204-212.

41. Hoeller S, Sperger A, Valenta C. Lecithin based nanoemulsions: $A$ comparative study of the influence of non-ionic surfactants and the cationic phytosphingosine onphysicochemical behaviour and skin permeation. Int. J. Pharm. 2009; 370: 181-186.

42. Coulmana SA, Anstey A, Gateleyb $C$, Morrisseyc $A$, McLoughlind C, Allendera C, Birchall JC. Microneedle mediated delivery of nanoparticles into human skin. Int. J. Pharm. 2009; 366: 190-200.

43. Ran S, Downes A, Thorpe PE. Increased exposure of anionic phospholipids on the surface of tumor blood vessels. Cancer Res. 2002; 62: 6132-6140.

44. Yang $R$, Han X, Shia, X, Cheng G, Shim Ch, Cui $F$ Cationic formulation of paclitaxel-loaded poly $D, L$ lactic-co-glycolic acid (PLGA) nanoparticles using an emulsion-solvent diffusion method. Asian $\mathrm{J}$. Pharmaceut. Sci. 2009; 4: 89-95.

45. Lee ES, Na K, Bae YH. Polymeric micelle for tumor $\mathrm{pH}$ and folate-mediated targeting. J. Control. Release. 2003; 91: 103-113.

46. Mahoney BP, Raghunand $N$, Baggett $B$, Gillies RJ. Tumor acidity, ion trapping and chemotherapeutics. I. Acid $\mathrm{pH}$ affects the distribution of chemotherapeutic agents in vitro. Biochem. Pharmacol. 2003; 66: 1207-1218.

47. Garcia-Martin ML, Martinez GV, Raghunand N, Sherry $A D$, Zhang $S$, Gillies $R J$. High resolution $p H e$ imaging of rat glioma using $\mathrm{pH}$-dependent relaxivity. Magn. Reson. Med. 2006; 55: 309-315.

48. Yang $R$, Shima WS, Cuic FD, Chengc $G$, Hanc $X$, Jin $Q R$, Kima $D D$, Chunga S, Shima CK. Enhanced electrostatic interaction between chitosan-modified PLGA nanoparticle and tumor. Int. J. Pharm. 2009; 371: 142-147.

49. Chen $X$, Wang $X$, Wang $Y$, Yang, L, Hu J, Xiao W, Fu A, Cai L, LiX, Ye X, Liu Y, Wu W, Shao X., Mao Y, Wei $Y$, Chen $L$. Improved tumor-targeting drug delivery and therapeutic efficacy by cationic liposome modified with truncated bFGF peptide. J. Control. Release 2010; 145: 17-25.

50. Sethuraman VA, Han Bae Y. TAT peptide-based micelle system for potential active targeting of anti-cancer agents to acidic solid tumors. J. Control. Release 2007; 118: 216-224.

51. Needham $D$, Ponce A. Nanoscale drug delivery vehicles for Solid tumors, Nanotechnology for cancer therapy, CRC Press, Taylor \& Francis; 2002; $p 677$.

52. Dubernet BC, Couvreur P. Nanoparticles in cancer therapy and diagnosis. Adv. Drug Deliver. Re., 2002; 54: 631-651.

53. Cafaggi S, Russo E, Stefani $R$, Leardi R, Caviglioli $G$, Parodi $B$, Bignardi $G$, Detotero $D$, Viale $M$. Preparation and evaluation of nanoparticles made of chitosan or N-trimethyl chitosan and a cisplatinalginate complex. J. Control. Release 2007; 121: 110-123.

54. Yang R, Yong SG, Shim WS, Cui F, Cheng G, Kim IW, Kim DD, Chung SJ, Shim CK. Lung-specific delivery of paclitaxel by chitosan-modified PLGA nanoparticles via transient formation of microaggregates. J. pharm Sci. 2009; 98: 970-984.

55. Chun ChJ, Lee SM, Kim S, Yang $H$, Song SC. Thermosensitive poly(organophosphazene)paclitaxel conjugate gels for antitumor applications. Biomaterials 2009; 30: 2349-2360.

56. Gregoriadis G. Liposomes technology, Volume III CRC Press, Taylor \& Francis; 2007. 156p.

57. Beduneau A, Saulnier P, Benoit JP. Active targeting of brain tumors using nanocarriers. Biomaterials 2007; 28: 4947-4967.

58. Park JH, Lee S, Kim J, Park K, Kim K, Kwon IC. Polymer nanomedicine for cancer therapy. Prog. Polym. Sci. 2008; 33: 113-137.

59. Kommareddy S, Shenoy DB, Amiji MM. Long-circulating polymeric nanoparticles for drug and gene delivery to tumors, Nano technology for cancer therapy, CRC Press, Taylor \& Francis; 2007; p 231.

60. Juillerat-Jeanneret $L$. The targeted delivery of cancer drugs across the blood-brain barrier: chemical modifications of drugs or drug-nanoparticles. Drug Discov. Today 2008; 13: 1099-1106.

61. Kaur IP, Bhandari R., Bhandari S, Kakkar V. Potential of solid lipid nanoparticles in brain targeting. J. Control Release 2008; 127: 97-109.

62. Parikh T, Bommana MM, Squillante E. Efficacy of surface charge in targeting pegylated nanoparticles of sulpiride to the brain. Eur. J. Pharm. Biopharm. 2010; 74: 442-450.

63. Chena $H$, Tanga I, Qina $Y$, Yina $Y$, Tanga J, Tanga $W$, Suna X, Zhanga Z, Liuc J, Hea Q. Lactoferrinmodified procationic liposomes as a novel drug carrier for brain delivery. Eur. J. Pharm. Sci. 2010; 40: 94-102.

64. Ku S, Yan F, Wang Y, Sun Y, Yang N, Ye L. The bloodbrain barrier penetration and distribution of PEGylated fluorescein-doped magnetic silica nanoparticles in rat brain. Biochem. Bioph. Res. Co. 2010; 394: 871-876.

65. Wanga $S$, Jianga $T$, Maa $M$, Hua $Y$, Zhangb J. Preparation and evaluation of anti-neuroexcitation peptide (ANEP) loaded N-trimethyl chitosan 
chloride nanoparticles for brain-targeting. Int. J. Pharmaceut. 2010; 386: 249-255.

66. Huang M, Khor E, Lim LY. Uptake and cytotoxicity of chitosan molecules and nanoparticles: effects of molecular weight and degree of deacetylation. Pharm. Res. 2004; 21: 344-353.

67. Amidi M, Romeijn SG, Verhoef JC, Junginger $H E$, Bungener L, Huckriede A, Crommelin DJA, Jiskoot W. N-Trimethyl chitosan (TMC) nanoparticles loaded with influenza subunit antigen for intranasal vaccination: biological properties and immunogenicity in a mouse model. Vaccine 2007; 25: 144-153.

68. Khdair A, Chen D, Patil Y, Ma L, Dou P, Shekhar M,Panyam J. Nanoparticle-mediated combination chemotherapy and photodynamic therapy overcomes tumor drug resistance. J. Control. Release 2010; 141: 137-144.

69. Lei $T$, Srinivasan $S$, Tang $Y$, Manchanda $R$, Nagesetty A, Fernandez-Fernandez A, McGoron AJ. Comparing cellular uptake and cytotoxicity of targeted drug carriers in 2 cancer cell lines with different drug resistance mechanisms. NanomedNanotechnol. 2011; 7: 324-332.

70. Zhang $Y$, Tang $L$, Sun $L$, Bao J, Song $C$., Huang $L$, Liu $K$, Tian Y, Tian G, Li Z, Sun $H$, Mei L. A novel paclitaxel-loaded poly(e-caprolactone)/Poloxamer 188 blend nanoparticle overcoming multidrug resistance for cancer treatment. Acta. Biomater. 2010; 6: 2045-2052. 